\title{
Robotic photosieving from low-cost multirotor sUAS: A proof-of-concept
}

\author{
P.E. Carbonnneau*1, S. Bizzi ${ }^{2}$ and G. Marchetti ${ }^{2}$
}

1: Department of Geography, Durham University, Durham, UK. Email:

patrice.carbonneau@durham.ac.uk

2: Department of Electronics, Information, and Bioengineering, Politecnico di Milano, Piazza Leonardo da Vinci, Milano, Italy.

*: Corresponding author

\section{Abstract}

Measurement of riverbed material grainsizes is now a routine part of fieldwork in fluvial geomorphology and lotic ecology. In the last decade, several authors have proposed remote sensing approaches of grain size measurements based on terrestrial and aerial imagery. Given the current rise of small Unmanned Aerial System (sUAS) applications in geomorphology, there is now increasing interest in the application of these remotely sensed grain size mapping methods to sUAS imagery. However, success in this area has been limited due to two fundamental problems: lack of constraint of image scale for sUAS imagery and blurring effects in sUAS images and resulting orthomosaics. In this work, we solve the former by showing that SfM-photogrammetry can be used in a direct georeferencing (DG) workflow (i.e. with no ground validation) in order to predict image scale within margins of $3 \%$. We then propose a novel approach of robotic photosieving of dry exposed riverbed grains that relies on near-ground images acquired from a low-cost sUAS and which does not require the presence of ground control points or visible scale objects. We demonstrate that this absence of scale objects does not affect photosieving outputs thus resulting in a low-cost and efficient sampling method for surficial grains.

\section{Keywords: sUAS, drone, grainsize mapping, fluvial remote sensing}

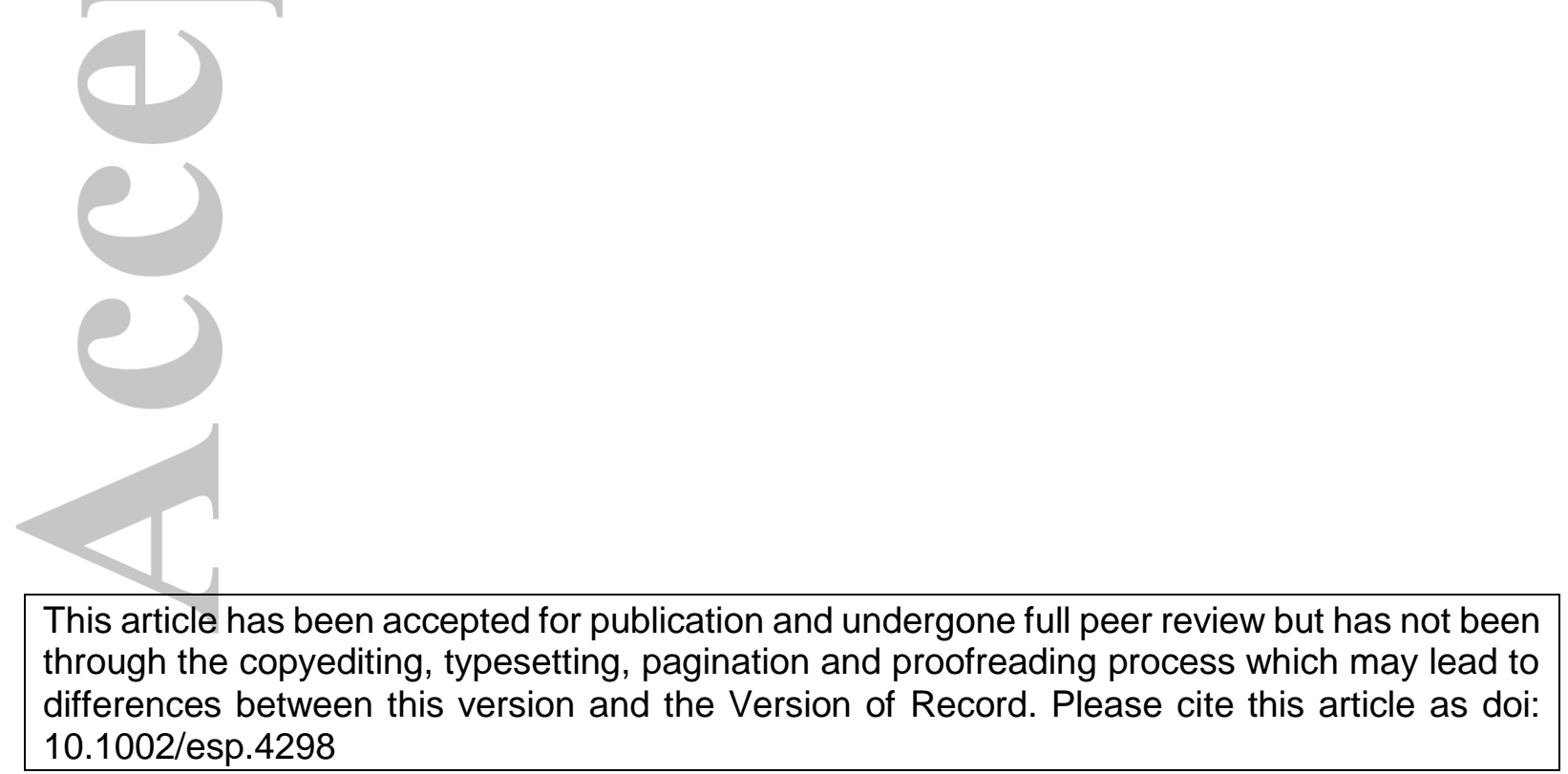

This article is protected by copyright. All rights reserved. 


\section{Introduction}

The remote sensing of riverbed material particle size has been the focus of a substantial amount of research in recent years e.g. (Buscombe, 2008; Buscombe et al., 2010; Buscombe and Masselink, 2009; Carbonneau, 2005; Carbonneau et al., 2005b, 2005a; Detert and Weitbrecht, 2012; Graham et al., 2010; Woodget and Austrums, 2017). Generally speaking these methods of particle size determination from imagery can be classified in two broad families: Object-based and statistical correlation approaches. As the name implies, the object-based approaches rely on visual or automated identification and measurement of clast objects in imagery. Early approaches, dubbed as 'photosieving', relied on visual clast identification and measurement in terrestrial photographs (Adams, 1979; Ibbeken and Schleyer, 1986). Alternatively, several authors have presented automated approaches which rely on object-based image analysis (OBIA) in order to segment clasts into separate objects that can be fitted to an ellipsoid in order to estimate both the a-axis and b-axis most commonly used in particle size descriptions e.g. (Butler et al., 2001; Detert and Weitbrecht, 2012; Graham et al., 2010, 2005). The second family of image-based grain-size measurement approaches moves away from individual clast identification and correlates the statistical properties of image patches with their median or average grain-size. Within this approach, several algorithms and statistical metrics have been applied such as image texture (Black et al., 2014; Carbonneau et al., 2005c, 2005a, 2004; Verdú et al., 2005), image autocorrelation (Buscombe and Masselink, 2009; Rubin, 2004) and wavelet transforms (Buscombe et al., 2010; Buscombe and Rubin, 2012). With the exception of Buscombe et al.(2010), a common limitation with these statistical correlation approaches is that they require empirical calibration data which is needed to establish a predictive relationship yielding grainsizes from some local image statistic. More generally, one common-point that links both the statistical correlation and object-based approach is that they all measure particle size in image pixel units and then, at some point in their respective processes, convert these dimensions to linear units via knowledge of image scale and/or pixel dimensions. In the case of terrestrial imagery, image scale is commonly established with the inclusion of a scale object in the photographs. In the case of airborne imagery acquired from manned aircraft, the nominal altitude above ground level is a closely controlled parameter which allows an accurate image scale to be calculated. However, in the case of drone imagery, precise altitude AGL (Above Ground Level) is not well constrained and as a result spatial resolution is not known a priori. Therefore, precise determination of image scale for each image in a small Unmanned Aerial System (sUAS) survey remains problematic.

In this short communication, we present a corollary of the work of Carbonneau and Dietrich (2017). These authors have demonstrated that a Direct Georeferencing (DG) approach with low-cost sUAS can accurately calibrate the camera focal length and predict that the scale of a survey site to within $c a . \pm 1 \%$. Here, we use these findings to demonstrate that individual image scale can also be strongly constrained with the proper use of SfM-Photogrammetry in a DG workflow and our results show that the altitude above ground level (AGL) and associated scale of images can be predicted to within $3 \%$ when they are included in a larger photogrammetric bloc which is georeferenced with a DG workflow (with no ground validation). From this foundation, we present the proof-of-concept for a new robotic photosieving approach applied here to dry exposed riverbed material. The objective of robotic photosieving is to sample riverbed grainsizes without the need for any type of field calibration data, it relies on non-overlapping, near-ground imagery acquired from sUAS at flight altitudes below $10 \mathrm{~m}$ AGL (Above Ground Level). Crucially, these images are also part of a larger 
photogrammetric bloc that includes higher altitude images with a strong network geometry. In such cases, the low-altitude imagery can be aligned in the overall photogrammetric bloc and their resulting position and spatial resolution precisely predicted from SfM-photogrammetry. The resulting $\mathrm{mm}$-scale imagery clearly resolves particle edges for fluvial gravels and cobbles and has further potential to resolve the gravel/sand transition. We argue that robotic photosieving, combined to recent low-cost multirotor sUAS, provides a novel way forward for remote grain size measurements that will transform current fluvial survey practices.

\section{Methods}

\section{Site Selection and Photosieving Data Acquisition}

Field experiments were conducted in Northern Italy on the rivers Po, and Sesia. Three sites were selected according to grain size: on the Sesia, we identified a very coarse site with a cobble/boulder bed. On the Po, we identified two sites: one with a sand bed and another with a fine to coarse gravel bed. At each site, we deployed a series of fifteen $30 \mathrm{~cm}$ rulers (total length of $310 \mathrm{~mm}$ ) in bright opaque colours as a testable reference scale for aerial imagery. The stability of ruler length was tested by sampling 15 rulers with digital callipers yielding an average length of $310.2 \mathrm{~mm}$ with a standard deviation of $1.1 \mathrm{~mm}$. We use $310 \mathrm{~mm}$ as a meaningful value for ruler length. Our approach involves SUAS imagery taken from a hovering position at very low altitudes (below $10 \mathrm{~m}$ ) and therefore requires a multirotor system. Imagery was acquired with a consumer-grade Phantom 4 sUAS manufactured by DJI inc. Take-off weight is $1.38 \mathrm{Kg}$ and it is equipped with a $6.17 \times 4.55 \mathrm{~mm}$ CMOS colour sensor acquiring 12Mpix still images. The camera has a stock focal length of $3.61 \mathrm{~mm}$ and is mounted on a gimbal which damps vibrations and compensates for rotational motions of the sUAS. The DJI Phantom 4 is also equipped with a series of downwards facing sensors designed to assist hover stability when at low altitudes $(<10 \mathrm{~m})$ or in GPS-deprived environments. The Visual Positioning System (VPS) uses a combination of ultrasound emitters and optical flow cameras to compensate for well-known lack of indoor reception and limitations in the vertical precision of consumer grade GPS receivers. For the surveys reported here, sUAS flight was automated with a third-party app: Litchi. This app gives the pilot full control on all flight parameters: Altitude, heading, velocity, intervals, camera angle and settings. The app also allows the pilot to enter and use preprogrammed waypoints in order to determine flight pathways. For each site, the fifteen rulers were dropped on the gravel bar and a powered-up drone was carried manually over the rulers. At each, a configured button on the pilot's remote control was used to add a waypoint in the piloting app in order to guide the sUAS back to the ruler. This was found to be the most effective method of entering the position of the ruler into the flight control app. Once the rulers were deployed and their position recorded, the waypoint altitudes were increased to $7 \mathrm{~m}$ in order to mitigate for the relatively low accuracy of the on-board GPS $( \pm 2.5 \mathrm{~m})$. Given that the return to any given waypoint relies on 2 measurements, one to set the waypoint and another to return, the expected circle of error for the waypoint navigation is in the order $10 \mathrm{~m}$. At $7 \mathrm{~m} \mathrm{AGL}$, and given the camera's field of view (FOV) of $94^{\circ}$ and 4:3 image ratio, the image footprint is $c a .15 \times 11 \mathrm{~m}$. The image dimensions therefore enclose the expected error thus allowing us to assume that the ruler will be captured in the image despite navigation errors. The resulting pixel resolution is $3.5 \mathrm{~mm}$. However, readers should note that the effective altitude above ground level (AGL) along with image footprint and resolution, will change as ground elevation varies and require accurate estimation. Figures 1 shows an example of a low-altitude image.

This article is protected by copyright. All rights reserved. 
We then proceed to design the photogrammetric survey that we hypothesize will allow us to predict the scale of the near-ground images. Readers should note that we make an important assumption: the method developed here is intended to work in tandem with sUAS mapping methods. Consequently, in what follows, we are designing the photogrammetric survey based on our experience in SfM-Photogrammetry as published in recent literature (Carbonneau and Dietrich, 2017; Fonstad et al., 2013; James and Robson, 2014). Our image geometry therefore aims to deliver best results, in a DG workflow, for camera calibration and topographic precision and also to deliver high quality orthoimagery and topography that are commonly expected as part of any sUAS survey. Carbonneau and Dietrich (2017) and Fonstad et al. (2013) both recommend the use of two flight altitudes, the drone was therefore programmed to collect imagery at altitudes of $20 \mathrm{~m}$ and $60 \mathrm{~m} \mathrm{AGL}$ along straight flight lines in multiple directions. For these 2 higher altitudes, waypoints were not used to define individual image acquisition locations, they were only used to determine the endpoints of individual flight lines. The relatively low altitude of $20 \mathrm{~m}$ was selected in order to minimise disparities between the near-ground imagery and the higher altitude imagery and therefore insure that the image matching algorithm in the photogrammetric software could find sufficient correspondence with the near-ground images. Images at altitudes of $20 \mathrm{~m}$ were acquired at nadir (camera axis normal to the surface). The higher altitude was set at $60 \mathrm{~m}$ since this is the legal limit for drone flights in Italian airspace operated by non-commercial pilots. These images were acquired at 30 degrees off-nadir. sUAS velocity and image intervals were set for $80 \%$ forward overlap. Lines at $20 \mathrm{~m} \mathrm{AGL}$ were positioned for $50 \%$ sidelaps with lines at $60 \mathrm{~m}$ perpendicular to these and providing additional overlap in the image network geometry. Given the flight time of the Phantom 4, we anticipated that this flight pattern could cover areas in excess of $70000 \mathrm{~m}^{2}$ and leave sufficient battery to collect the near-ground imagery required for the photosieving.

Another key point that readers should note is that there is no spatial overlap within the near-ground image data. Whilst it would be possible to acquire overlapping images within a full photogrammetric survey with a base altitude of $7 \mathrm{~m}$, the low ground altitude, combined to the requirement of $80 \%$ forward overlap and 50-60\% sidelap in the imagery, would lead to forward velocities of only $3 \mathrm{~km} / \mathrm{h}$ and flight lines separated by $\sim 5 \mathrm{~m}$. For example, with a flight time of 20 minutes, the Phantom 4 could cover 1 linear $\mathrm{km}$ under these conditions. At $7 \mathrm{~m}$ altitude the image footprint is $11 \mathrm{~m}$. If we choose 2 lines of $500 \mathrm{~m}$, separated by $5 \mathrm{~m}$, we obtain a total image swath of $500 \mathrm{mX17} \mathrm{m}$ which is only $8500 \mathrm{~m}^{2}$. We consider this working area to be unacceptably small and we therefore opted for a sampling approach where any given near-ground photo with $\mathrm{mm}$-scale resolution only overlaps with higher altitude photos with $\mathrm{cm}$-scale resolutions. Therefore, the photogrammetric process described here cannot deliver mm-scale topographic data based on the resolution of the near-ground imagery. For the near-ground imagery, we only aim to derive the accurate position of the camera at the time of acquisition and then use this information in the calculation of an accurate image scale.

\section{Photogrammetric Processing}

Image networks for each site were processed as single 'chunks' (i.e. image blocs) with Agisoft Photoscan version 1.2.6. First we setup 3 chunks which had data for each of 3 sites at all altitudes. Second, we setup 3 additional chunks where the $60 \mathrm{~m}$ altitude data was excluded. This data will therefore be processed without oblique views. Photoscan was used to align the image network, on 
the 'highest' accuracy setting and build a dense point cloud on the 'medium' setting. Raster DEMs are not used in this work. For additional detail on the steps used in SfM-Photogrammetry, readers can now consult a growing body of published work (Bemis et al., 2014; Fonstad et al., 2013; James and Robson, 2012; Smith et al., 2016; Westoby et al., 2012). The image blocs were referenced solely from the geotags recorded in the image metadata. Carbonneau and Dietrich (2017) found that such an approach can yield high quality data with slope errors below 1 degree and scale errors $0.4-4 \%$. The photogrammetric processing had 4 key outputs: point clouds of the bar surfaces, point clouds of the adjusted camera positions, adjusted focal lengths and undistorted photos. The 'adjusted' camera positions correspond to the final positions and orientations after a least-squares bundle adjustment implemented by Photoscan. These camera positions will be more reliable than the raw GPS metatags present in the imagery. During the bundle adjustment, Photoscan also estimates camera parameters in order to refine and adjust values for lens distortion and focal length. Finally, we used Photoscan to export undistorted (non-orthorectified) imagery. The point cloud and camera location data was then imported into CloudCompare. In accordance with the findings of Carbonneau and Dietrich (2017), we assume that the best quality surfaces are those processed with all 3 altitudes and we use these as the reference surfaces for each site. TIN meshes were then calculated as the bestfitting plane spanning point trios for each surface. Once these meshes were calculated, we imported the $\mathrm{XYZ}$ adjusted camera positions as a point cloud and calculated a cloud-to-mesh distance that yielded the distance of each camera to the interpolated mesh surface along the Z-direction of the model. We thus avoid the interpolation effects associated to raster DEMs and limit the role of interpolation to the calculation of a mesh for the reference surface.

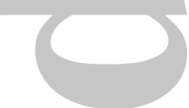

\section{Remote Scale Predictions}

After point cloud generation and processing, we proceeded to scale predictions. Our approach is to predict the length of the rulers in the near-ground imagery with a combination of camera and flight altitude data and to compare these predictions to the known ruler length of $310 \mathrm{~mm}$. Using basic camera geometry, the length of a ruler in object space (i.e. real-world space) can be predicted by eq. 1:

$$
R_{o}=\frac{H * R_{i}}{f}
$$

Where $R_{0}$ is the length of the ruler in object space, $H$ is the flying height expressed in linear units and calculated as the height difference between the reference surface and the camera position, $R_{i}$ is the length of the ruler in image space as measured in the imagery and expressed as a number of image pixels, we used the Matlab imtools function to display and measure ruler length on-screen. $f$ is the focal length expressed in pixel units. We test 3 focal lengths: the stock focal length supplied by DJI (Case 1); the single-altitude (with only images acquired at $20 \mathrm{~m} \mathrm{AGL}$ ) calibrated focal length (Case 2) and the double-altitude (with images from both 20 and $60 \mathrm{~m} \mathrm{AGL}$ ) calibrated focal length (Case 3).

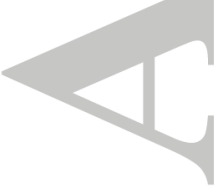


Finally, we examine how errors in scale predictions would propagate into a photosieving project composed of multiple images. Images were classified with an object-based approach in ENVI 5.3 which allowed us to quantify sand $\%$ by surface. Images with more than a $20 \%$ presence (by surface) of sand were excluded from further analysis since $\mathrm{mm}$-scale imagery is not capable of delineating individual sand grains. We processed the remaining images (27) with BASEGRAIN, an open-source package for automated object-based grainsize measurement (Detert and Weitbrecht, 2012). Given the spatial resolution of the images, the cut-off b-axis length was set to $10 \mathrm{~mm}$ in order to exclude the detection of any grains smaller that $c a$. $3 X 3$ pixels. BASEGRAIN parameters were saved and for each of the 27 images, the photosieving process was run twice. The first time, the scale was manually determined by re-measuring the ruler object on-screen with the BASEGAIN 'scale' tool and setting this length to $310 \mathrm{~mm}$. Individual detected clasts from all images were then merged to produce 1 large population of photosieved grains. The second time, the image scale was directly entered as that predicted by the case $3 \mathrm{SfM}$-photogrammetry workflow, the resulting clast data was once again merged to produce a second large population of sampled grains. Outputs were analysed by comparing grain size fractions and by performing a statistical Wilcoxon rank sum test to verify if the median grain sizes of both large distributions were statistically equal. Figure 2 presents a flowchart summarising the entire robotic photosieving workflow.

\section{Results}

Table 1 gives the basic descriptors of the photogrammetry outputs produced for each of the three sites. A key result is that Photoscan failed to calibrate the focal length without oblique view images (case 2) and returned the stock value as the adjusted focal length. Case 2 results are therefore not kept for further analysis. Model examination revealed that 32 of the 45 near ground images yielded usable data. Three near-ground image acquisitions missed their target ruler. Clearly in these cases, the GPS error exceeded the error of $\pm 2.5 \mathrm{~m}$ in XY reported by DJI. The likely explanation for this is simply that these position samples are from the tail of the distribution used by the manufacturer to establish an RMS error value to be passed to the consumer. For near-ground images, the average position difference before and after the photogrammetric adjustment is $-0.20 \mathrm{~m},-0.14 \mathrm{~m}, 2.25 \mathrm{~m}$, in $\mathrm{X}$, $\mathrm{Y}$ and $\mathrm{Z}$ respectively. However, the more significant issue was that 10 near-ground images did not align in the overall photogrammetric models thus not returning an adjusted camera position and making an accurate determine of altitude AGL impossible. Seven of these images were for the sandy site on the river Po. This suggests that matching failure is due to a lack of unique key point features identifiable in both near-ground and higher altitude imagery.

Inspection of the camera altitudes AGL shows that the flight altitudes AGL as estimated from the image alignment varied from $3.8 \mathrm{~m}$ to $7.4 \mathrm{~m}$. Figure 3 shows altitude distribution. Equation 1 was then applied to the resulting flight altitudes and using the focal length data presented in table 1 . The prediction errors were then calculated as a $\%$ of actual ruler length. Figure 4 shows results with mean scale predictions errors of $-0.4 \%$ and $-1.2 \%$ with standard deviations of $1.7 \%$ and $2.6 \%$ respectively. Maximum errors were $-5.11 \%$ for the stock focal (case $1 / 2$ ) and $-4.03 \%$ for the calibrated focal length (case 3 ). Calibration of the focal length did not improve the mean error, but it reduced standard deviation and produced a normal distribution of the errors. Overall these results are coherent with previous findings. Carbonneau and Dietrich (2017) found that using a direct 
georeferencing workflow could restitute model scales to within $0.2-4 \%$ depending on the image network geometry. Furthermore, James et al (2017) propose a simple relationship of $\mathrm{n}^{-1 / 2} \mathrm{x}$ control precision as an estimate of absolute precision for an SfM survey. Using this relationship with our data (see table 1 ) we obtain precisions of $\sim 15 \mathrm{~cm}$. When compared to the planned flying height of $7 \mathrm{~m}$, this precision represents an error of $\sim 3 \%$ which very closely matches the finding above.

Initial observation of the low-altitude imagery clearly shows recognisable grains that are easily identified by a human observer (e.g. fig. 1). This indicates that aerial photosieving will be possible in analogy with the approach of Dugdale et al. (2010). Figure 5 displays a sample outputs from BASEGRAIN. We find that for sandy patches the object-based algorithm performed poorly with small homogenous patches of sand being falsely identified as clasts (top right of figure 5). Performance for larger clasts is good with most particles being successfully delineated. Figure 6 shows the results of the wider application of BASEGRAIN to a set of 27 images. Automated photosieving returned in excess of 1 million detected grains in the image dataset. Figure 6 shows that the distributions and resulting values for $D_{16}, D_{50}$ and $D_{84}$ are quasi-identical. Furthermore, the Wilcoxon rank sum test for equal medians (equivalent to a Mann Whitney $\mathrm{U}$-test) returned a $\mathrm{p}$-value of 0.71 therefore accepting the null hypothesis that the medians $\left(D_{50}\right)$ are equal.

\section{Discussion}

We have demonstrated that robotic photosieving using image scales predicted from SfMphotogrammetry delivers statistically equal grain size median values $\left(D_{50}\right)$ when compared to the traditional approach of determining spatial resolution with a visible scale object. Robotic photosieving can now be deployed without the presence of rulers meaning that the pilot and field crew do not need direct access to the sample location. Assuming most users will require 2D orthoimagery and 3D models, robotic photosieving only requires 1 additional flight when combined to the sUAS mapping approach used in Carbonnneau and Dietrich (2017). Our results have also again demonstrated the importance of oblique views for camera calibration. Based on our experience and results, we recommend that readers use multiple flight altitudes and viewing angles during surveys (Carbonneau and Dietrich, 2017; James et al., 2017).

Overall, the errors associated to robotic photosieving will match the widely researched errors already reported in the photosieving literature (Adams, 1979; Butler et al., 2001; Detert and Weitbrecht, 2012; Dugdale et al., 2010). Dugdale et al. (2010) present a summary of 11 additional papers reporting on the quality of photosieving results when compared to bulk sampling. Overall, authors report very strong linear relationships $\left(R^{2}>0.85\right)$ between photosieving and field outputs. This body of work also reports systematic errors ranging from $0.05 \psi$ to $0.33 \psi\left(1 \psi\right.$ unit $=\log _{2}(\mathrm{~mm}$ units)). Of specific interest to our results, Dugdale et al. (2010) also found that when using aerial images acquired from a manned aircraft for photosieving there was a slight positive bias of 1-2 pixels caused by operator errors when visually identifying particle edges. If transposed to our results, this would imply a bias of $4-6 \mathrm{~mm}$. The effect of this bias on the lower size fractions will be mitigated by our grain size cut-off of $10 \mathrm{~mm}$. However, given the importance of fine grain size fractions in many ecological processes such as fish habitat selection, this does impose a significant limitation to robotic photosieving. A further mitigation can be added by using a lower flying altitude for the robotic photosieving imagery. In the case of this proof-of-concept, the photosieving images were collected at an altitude of $7 \mathrm{~m}$ in order to offset the low precision of the on-board GPS and maximise the 
chances that near-ground images actually capture the ruler. This resulted in image spatial resolutions ranging from 2-3 $\mathrm{mm}$. This is a relatively low spatial resolution and a field operator with a camera could easily capture images with sub-mm spatial resolution. But, flight design can also improve the resolution, and given that rulers are not required for the actual implementation of robotic photosieving, the flying altitude can be set as low as 2-3 $m$ to mitigate the bias observed by Dugdale et al. (2010). However, readers should be cautioned that when operating at lower altitudes, photogrammetric errors can represent a greater percentage of the flying height and thus increase the errors in the robotic photosieving outputs. This could be mitigated by following the advice of James et al (2017) and increasing the number of images. For example, in the case of the present survey, table 1 shows that the predicted precision $p$, is on the order of $15 \mathrm{~cm}$ which is roughly $3 \%$ of the $7 \mathrm{~m}$ flying height. If we had collected the photosieving imagery at $2 \mathrm{~m}$, the error would have increased to $8 \%$. However, if we had collected twice as many images ( 500 ), the error could be reduced to $5 \%$. Alternatively, developing new low-cost technologies that improve the location accuracy of on-board drone positioning would also help improve the quality of robotic photosieving (James et al., 2017). However, it should be noted that by flying at lower altitudes, the correspondence between the near-ground imagery and the mid-altitude imagery will be reduced which might lead to a failure to align the photosieving images in the overall photogrammetric bloc. In such cases, the recommended solution is to reduce the altitude of the intermediate flight.

The approach presented here will facilitate the sampling and recording of fluvial grain size as well as the overall monitoring of gravel bar evolution. Similarly to geological work in Bemis et al.(2014), the geolocated grain size data combined to the high resolution photogrammetric surveys collected in the robotic photosieving workflow provide a recorded dataset that is not only useful for single-epoch analysis, but that will also prove valuable for change detection studies or simply when site information needs to be revisited by other researchers. Indeed, robotic photosieving can be used to rapidly collect a very large sample of surficial grain size. A typical drone battery of 15-20 minutes could easily provide in excess of 100 samples over areas of 1 hectare. Since Carbonneau et al. (2004) used a photosieving approach to calibrate their airborne grain size mapping method, robotic photosieving could also calibrate statistical grain size mapping methods if spatially continuous grain size information is required. However, we note that if spatially continuous grain size maps are not required, users may not actually need to undertake the extra step of calibrating and executing a grain size mapping algorithm and the robotic photosieving data could suffice for bar-scale grain size characterisation easily repeated on multiple gravel bars

Another key advantage of the robotic photosieving workflow described here is the potential to identify the gravel/sand transition identified by many authors as critical to ecohydraulic processes e.g. (Sear, 1993; Soulsby et al., 2001; Wu, 2000). If, as suggested above, robotic photosieving is carried out with a Phantom 4 drone at an altitude of $2 \mathrm{~m}$, then the resulting imagery would have a resolution of $1.1 \mathrm{~mm}$. If the same survey is carried out with a Phantom 4 pro and the improved camera, the resulting ground resolution would be $0.7 \mathrm{~mm}$. As we approach sub- $\mathrm{mm}$ scale imagery, we can begin to identify grains in the $2-5 \mathrm{~mm}$ range and thus identify the gravel/sand transition at 2 $\mathrm{mm}$ according to the Wentworth scale. Currently, ground-based imagery collected on foot is the only option for the acquisition of sub-mm imagery. Robotic photosieving therefore has the capacity to effectively automate the important field task of grain size sampling. 


\section{Conclusion}

We have presented the proof-of-concept for a novel approach to robotic measurement of dry, exposed, riverbed grains based on low-cost multirotor sUAS, that does not require any ground validation. Based on the prediction of image scale in a direct georeferencing (DG) SfM-

photogrammetry workflow, robotic photosieving will allow fluvial geomorphologist to rapidly sample particle size in reach-scale, inaccessible, areas with minimal field-effort. Furthermore, the use of a low-cost sUAS and one of many widely available SfM-photogrammetry packages, both commonly found in most geography departments, makes the process easily transferable to any researcher.

\section{Acknowledgments}

This work was art of the AMBER project. This project has received funding from the European Union's Horizon 2020 research and innovation programme under grant agreement No 689682. We would also like to thank Dr. Andrea Cominola for their assistance in the field. We thank Dr Martin Detert for installation help with BASEGRAIN. We also thank 2 anonymous reviewers and Dr Mike James for their constructive and productive comments.

\section{References}

Adams, J., 1979. Gravel size analysis from photographs, in: Journal of the Hydraulics Division, American Society of Civil Engineers. pp. 1247-1255.

Bemis, S.P., Micklethwaite, S., Turner, D., James, M.R., Akciz, S., Thiele, S.T., Bangash, H.A., 2014. Ground-based and UAV-Based photogrammetry: A multi-scale, high-resolution mapping tool for structural geology and paleoseismology. Journal of Structural Geology 69, Part A, 163178. doi:10.1016/j.jsg.2014.10.007

Black, M., Carbonneau, P., Church, M., Warburton, J., 2014. Mapping sub-pixel fluvial grain sizes with hyperspatial imagery. Sedimentology 61, 691-711.

Buscombe, D., 2008. Estimation of grain-size distributions and associated parameters from digital images of sediment. Sedimentary Geology 210, 1-10.

Buscombe, D., Masselink, G., 2009. Grain-size information from the statistical properties of digital images of sediment. Sedimentology 56, 421-438.

Buscombe, D., Rubin, D.M., 2012. Advances in the simulation and automated measurement of wellsorted granular material: 2. Direct measures of particle properties. Journal of Geophysical Research: Earth Surface 117. doi:10.1029/2011jf001975

Buscombe, D., Rubin, D.M., Warrick, J.A., 2010. A universal approximation of grain size from images of noncohesive sediment. Journal of Geophysical Research: Earth Surface 115. doi:10.1029/2009jf001477

Butler, J.B., Lane, S.N., Chandler, J.H., 2001. Automated extraction of grain-size data from gravel surfaces using digital image processing. Journal of Hydraulic Research 39, 519-529.

Carbonneau, P.E., 2005. The threshold effect of image resolution on image-based automated grain size mapping in fluvial environments. Earth Surface Processes and Landforms 30, 16871693.

Carbonneau, P.E., Bergeron, N., Lane, S.N., 2005a. Automated grain size measurements from airborne remote sensing for long profile measurements of fluvial grain sizes. Water Resources Research 41. doi:10.1029/2005wr003994 
Carbonneau, P.E., Bergeron, N.E., Lane, S.N., 2005b. Texture-based image segmentation applied to the quantification of superficial sand in salmonid river gravels. Earth Surface Processes and Landforms 30, 121-127.

Carbonneau, P.E., Bergeron, N.E., Lane, S.N., 2005c. Texture-based image segmentation applied to the quantification of superficial sand in salmonid river gravels. Earth Surface Processes and Landforms 30, 121-127.

Carbonneau, P.E., Dietrich, J.T., 2017. Cost-effective non-metric photogrammetry from consumergrade sUAS: implications for direct georeferencing of structure from motion photogrammetry. Earth Surface Processes and Landforms 42, 473-486.

Carbonneau, P.E., Lane, S.N., Bergeron, N.E., 2004. Catchment-scale mapping of surface grain size in gravel bed rivers using airborne digital imagery. Water resources research 40. doi:10.1029/2003wr002759

Detert, M., Weitbrecht, V., 2012. Automatic object detection to analyze the geometry of gravel grains-a free stand-alone tool, in: River Flow. pp. 595-600.

Dugdale, S.J., Carbonneau, P.E., Campbell, D., 2010. Aerial photosieving of exposed gravel bars for the rapid calibration of airborne grain size maps. Earth Surface Processes and Landforms 35, 627-639.

Fonstad, M.A., Dietrich, J.T., Courville, B.C., Jensen, J.L., Carbonneau, P.E., 2013. Topographic structure from motion: a new development in photogrammetric measurement. Earth Surface Processes and Landforms 38, 421-430.

Graham, D.J., Reid, I., Rice, S.P., 2005. Automated sizing of coarse-grained sediments: imageprocessing procedures. Mathematical Geology 37, 1-28.

Graham, D.J., Rollet, A.-J., Piégay, H., Rice, S.P., 2010. Maximizing the accuracy of image-based surface sediment sampling techniques. Water Resources Research 46. doi:10.1029/2008WR006940

Ibbeken, H., Schleyer, R., 1986. Photo-sieving: A method for grain-size analysis of coarse-grained, unconsolidated bedding surfaces. Earth Surface Processes and Landforms 11, 59-77.

James, M.R., Robson, S., 2014. Mitigating systematic error in topographic models derived from UAV and ground-based image networks. Earth Surf. Process. Landforms 39, 1413-1420. doi:10.1002/esp.3609

James, M.R., Robson, S., 2012. Straightforward reconstruction of 3D surfaces and topography with a camera: Accuracy and geoscience application. Journal of Geophysical Research: Earth Surface 117. doi:doi: 10.1029/2011JF002289

James, M.R., Robson, S., Smith, M.W., 2017. 3-D uncertainty-based topographic change detection with structure-from-motion photogrammetry: precision maps for ground control and directly georeferenced surveys. Earth Surface Processes and Landforms 42, 1769-1788. doi:10.1002/esp.4125

Rubin, D.M., 2004. A simple autocorrelation algorithm for determining grain size from digital images of sediment. Journal of Sedimentary Research 74, 160-165.

Sear, D.A., 1993. Fine sediment infiltration into gravel spawning beds within a regulated river experiencing floods: Ecological implications for salmonids. Regul. Rivers: Res. Mgmt. 8, 373390. doi:10.1002/rrr.3450080407

Smith, M.W., Carrivick, J.L., Quincey, D.J., 2016. Structure from motion photogrammetry in physical geography. Progress in Physical Geography 40, 247-275.

Soulsby, C., Malcolm, I. a., Youngson, A. f., 2001. Hydrochemistry of the hyporheic zone in salmon spawning gravels: a preliminary assessment in a degraded agricultural stream. Regul. Rivers: Res. Mgmt. 17, 651-665. doi:10.1002/rrr.625

Verdú, J.M., Batalla, R.J., Martínez-Casasnovas, J.A., 2005. High-resolution grain-size characterisation of gravel bars using imagery analysis and geo-statistics. Geomorphology 72, 73-93.

This article is protected by copyright. All rights reserved. 
Westoby, M.J., Brasington, J., Glasser, N.F., Hambrey, M.J., Reynolds, J.M., 2012. 'Structure-fromMotion'photogrammetry: A low-cost, effective tool for geoscience applications.

Geomorphology 179, 300-314.

Woodget, A.S., Austrums, R., 2017. Subaerial gravel size measurement using topographic data derived from a UAV-SfM approach. Earth Surface Processes and Landforms 42, 1434-1443. doi:10.1002/esp.4139

Wu, F.-C., 2000. Modeling embryo survival affected by sediment deposition into salmonid spawning gravels: Application to flushing flow prescriptions. Water Resour. Res. 36, 1595-1603.

doi:10.1029/2000WR900021
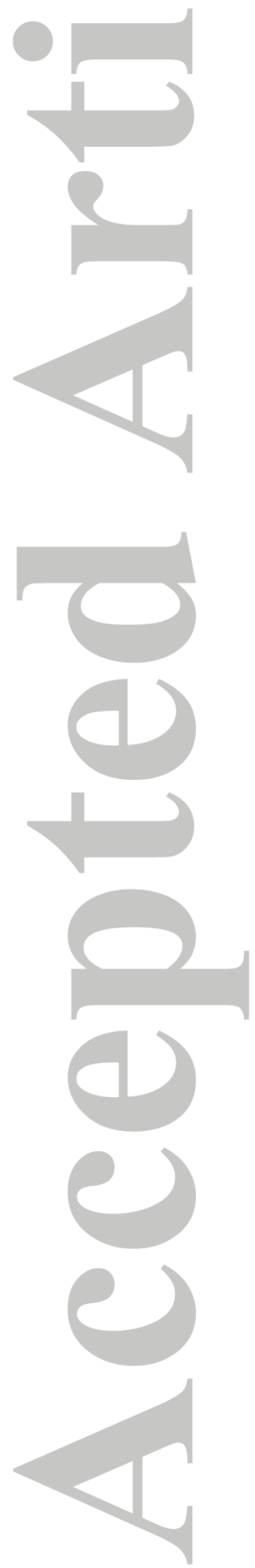

This article is protected by copyright. All rights reserved. 


\begin{tabular}{|l|l|l|l|l|l|l|l|l|l|}
\hline & $\begin{array}{l}\# \\
\text { Images }\end{array}$ & $\begin{array}{l}\text { Area } \\
{\left[\mathrm{m}^{2}\right]}\end{array}$ & $\begin{array}{l}\text { Pts } \\
{\left[\times 10^{3}\right]}\end{array}$ & $\begin{array}{l}\varepsilon \\
{[\mathrm{mm}]}\end{array}$ & $\begin{array}{l}p \\
{[\mathrm{~cm}]}\end{array}$ & $\begin{array}{l}R e \\
{[\mathrm{pix}]}\end{array}$ & $\begin{array}{l}\text { Focal [mm] } \\
\text { (case 1) }\end{array}$ & $\begin{array}{l}\text { Focal [mm] } \\
\text { (case 2) }\end{array}$ & $\begin{array}{l}\text { Focal [mm] } \\
\text { (case 3) }\end{array}$ \\
\hline Site 1 & 272 & 72423 & 586 & 20 & 15.16 & 0.65 & 3.610 & 3.610 & 3.651 \\
\hline Site 2 & 276 & 99375 & 624 & 20 & 15.05 & 0.84 & 3.610 & 3.610 & 3.536 \\
\hline Site 3 & 181 & 39274 & 529 & 20 & 18.58 & 1.47 & 3.610 & 3.610 & 3.701 \\
\hline
\end{tabular}

Table 1. Basic descriptors for photogrammetry outputs. The number of images includes the 15 near-ground photosieving images taken at each site. Site area is calculated by exporting a raster DEM at resolutions of $25 \mathrm{~cm}$ and summing the number of non-null pixel areas. $\varepsilon$ gives the expected relative noise error estimated from the results of Carbonneau and Dietrich (2017) as $\mathbf{0 . 1 \%}$ of the base flying height $(20 \mathrm{~m}) . p$ is the absolute precision as estimated by the work of James et al (2017). These authors show that $\left(\mathrm{n}^{-1 / 2} \mathrm{x}\right.$ control precision) is a good estimate for the absolute precision of a sUAS survey. Here $n$ is the number of points (here the number of images) and the control precision is taken as the reported GPS error of $\pm 2.5 \mathrm{~m} R e$ is the average reprojection error, in pixels, resulting from the alignment process. Case 1 focal length is the stock value provided by DJI inc. Case $\mathbf{2}$ is the focal length for model camera calibrations where $60 \mathrm{~m}$ flight data (oblique view) was excluded and Case $\mathbf{3}$ is the calibrated focal length with the full image dataset. Readers will notice that case $\mathbf{1}$ and case 2 focal lengths are identical. Photoscan failed to calibrate the focal length in case 2 and returned the stock value, all the solutions were processed with a full set of default camera parameters (focal, principal point offset and distortion).

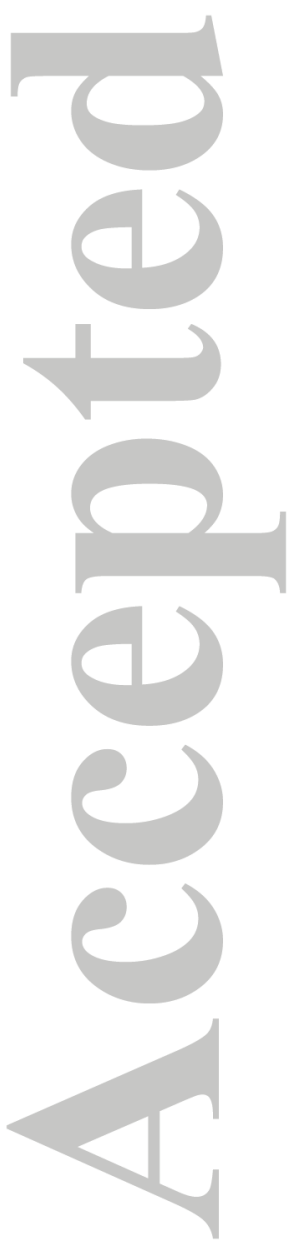

This article is protected by copyright. All rights reserved. 


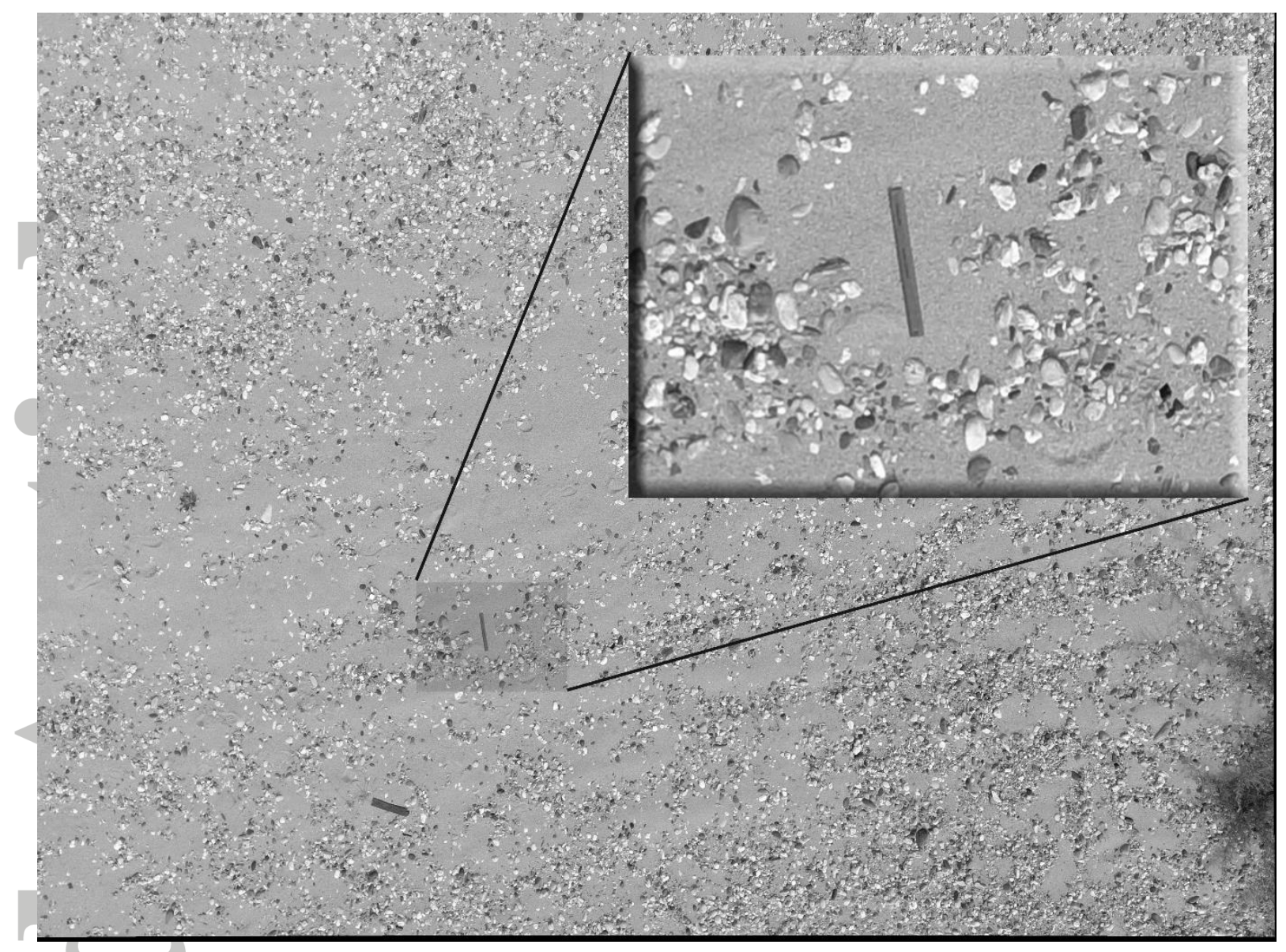

Figure 1: Sample photo of a ruler (total length $310 \mathrm{~mm}$ ) taken on the river Pô, Northern Italy.

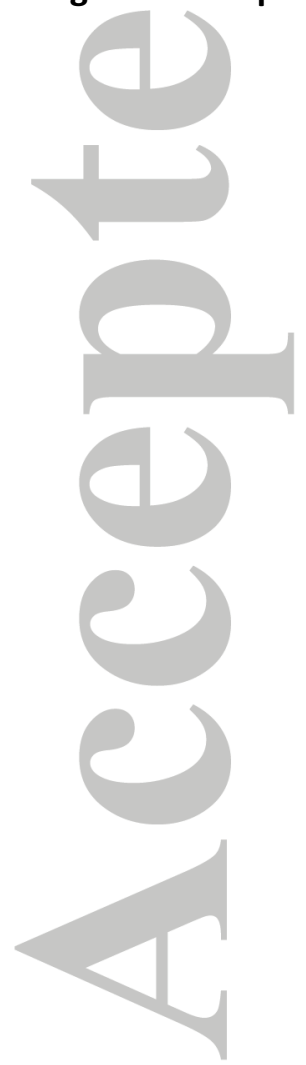

This article is protected by copyright. All rights reserved. 


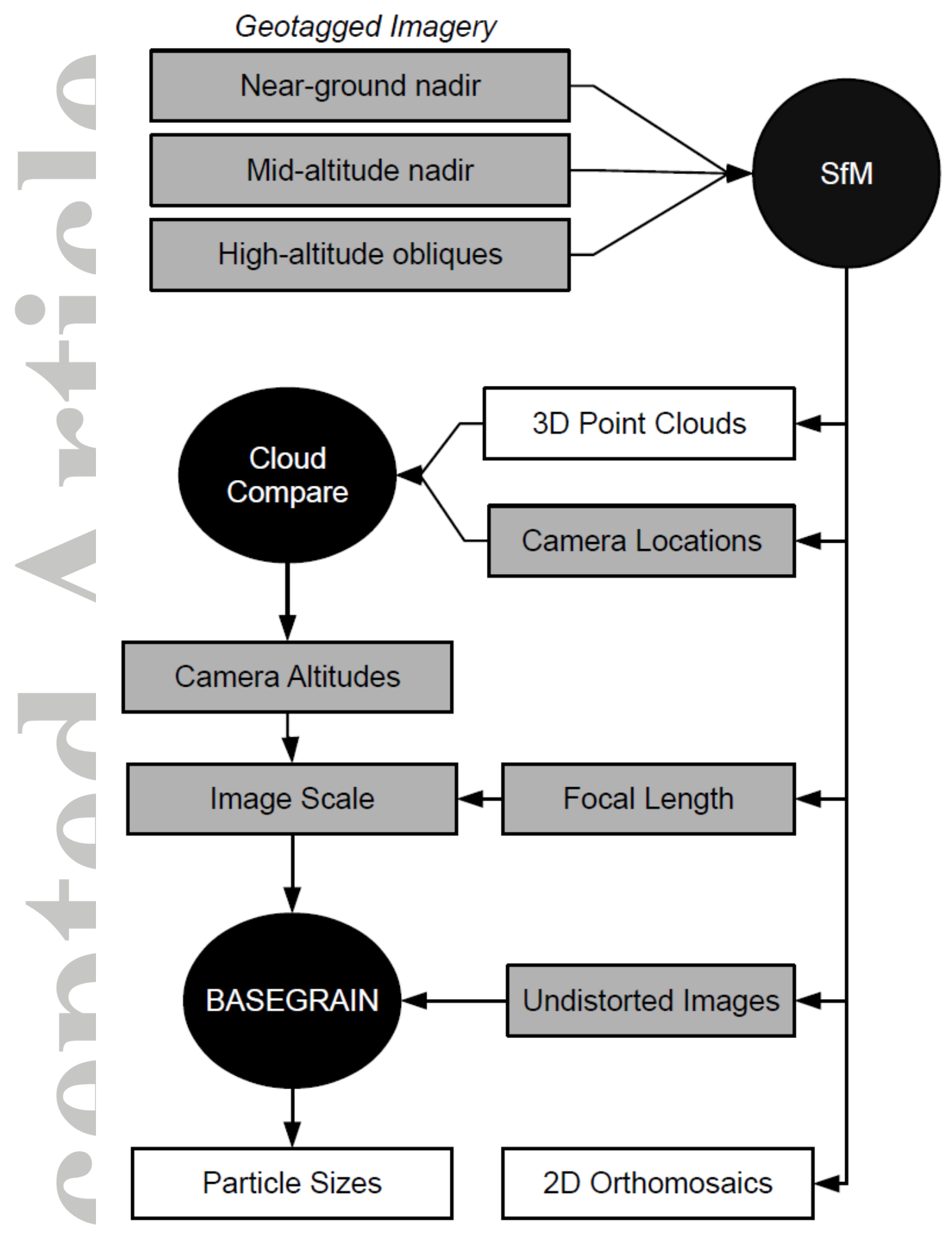

Figure 2. SUAS mapping and robotic photosieving flowchart. Grey elements are input or intermediary data. Black elements are processes requiring software. White elements are final outputs. 

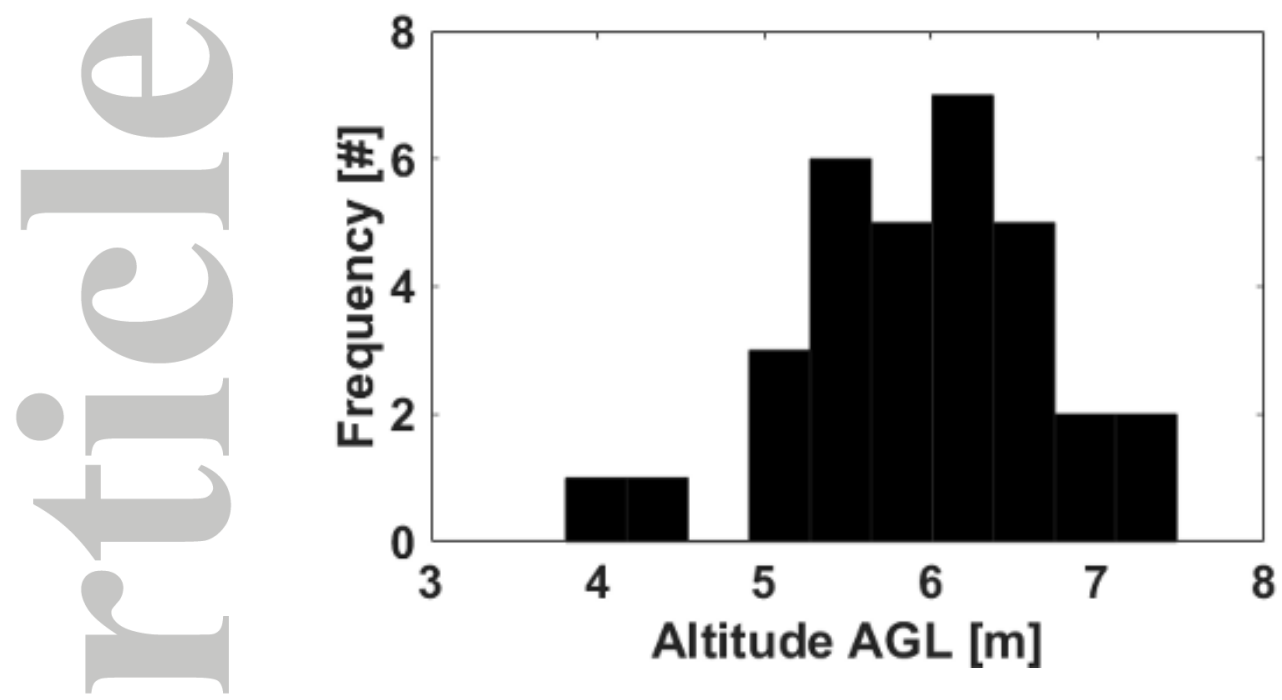

Figure 3. Camera altitude distribution for all sites. The variation around the programmed flight altitude of $7 \mathrm{~m}$ reflects both local changes in topography at the field sites and errors of the flight computer when trying to maintain altitude. For low altitudes AGL and where the Visual Positioning System functions to enhance GPS-Z precision, DJI reports these as $\pm 0.5 \mathrm{~m}$. Average difference of camera location in $\mathrm{Z}$ before and after photogrammetric adjustment, $2.25 \mathrm{~m}$, suggests this error may be higher. 
Stock Focal

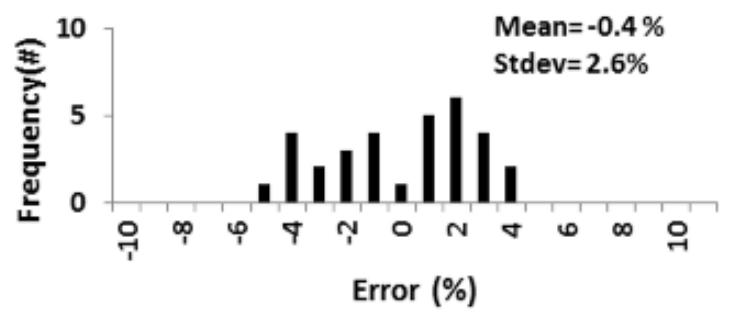

Adjusted Focal

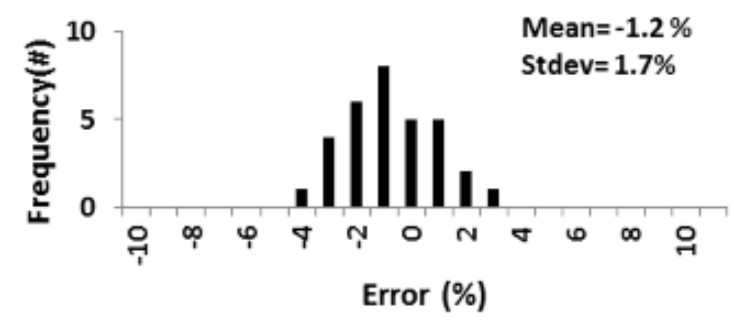

Figure 4 Ruler Length error distributions for stock (case 1) and adjusted (case 3 ) focal lengths. Error $\%$ was calculated with respect to actual ruler size of $310 \mathrm{~mm}$. Negative errors should be interpreted as the predicted length being smaller than the actual length.

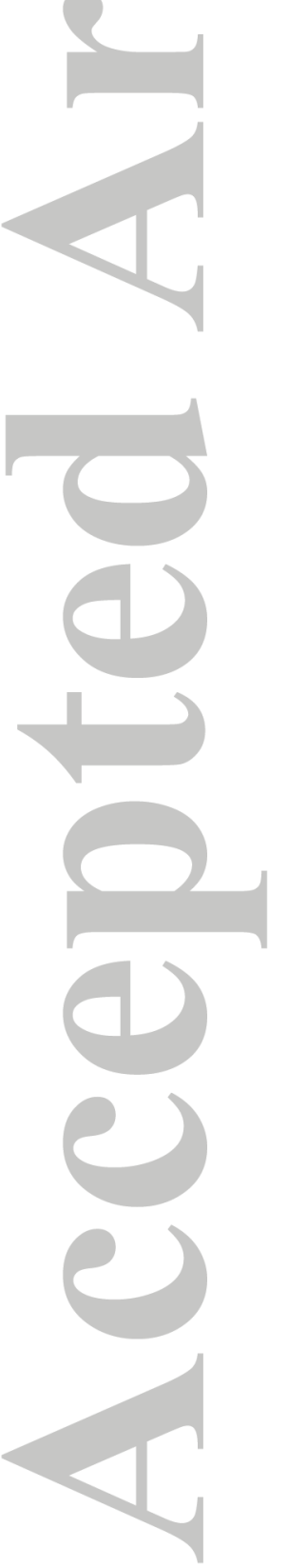




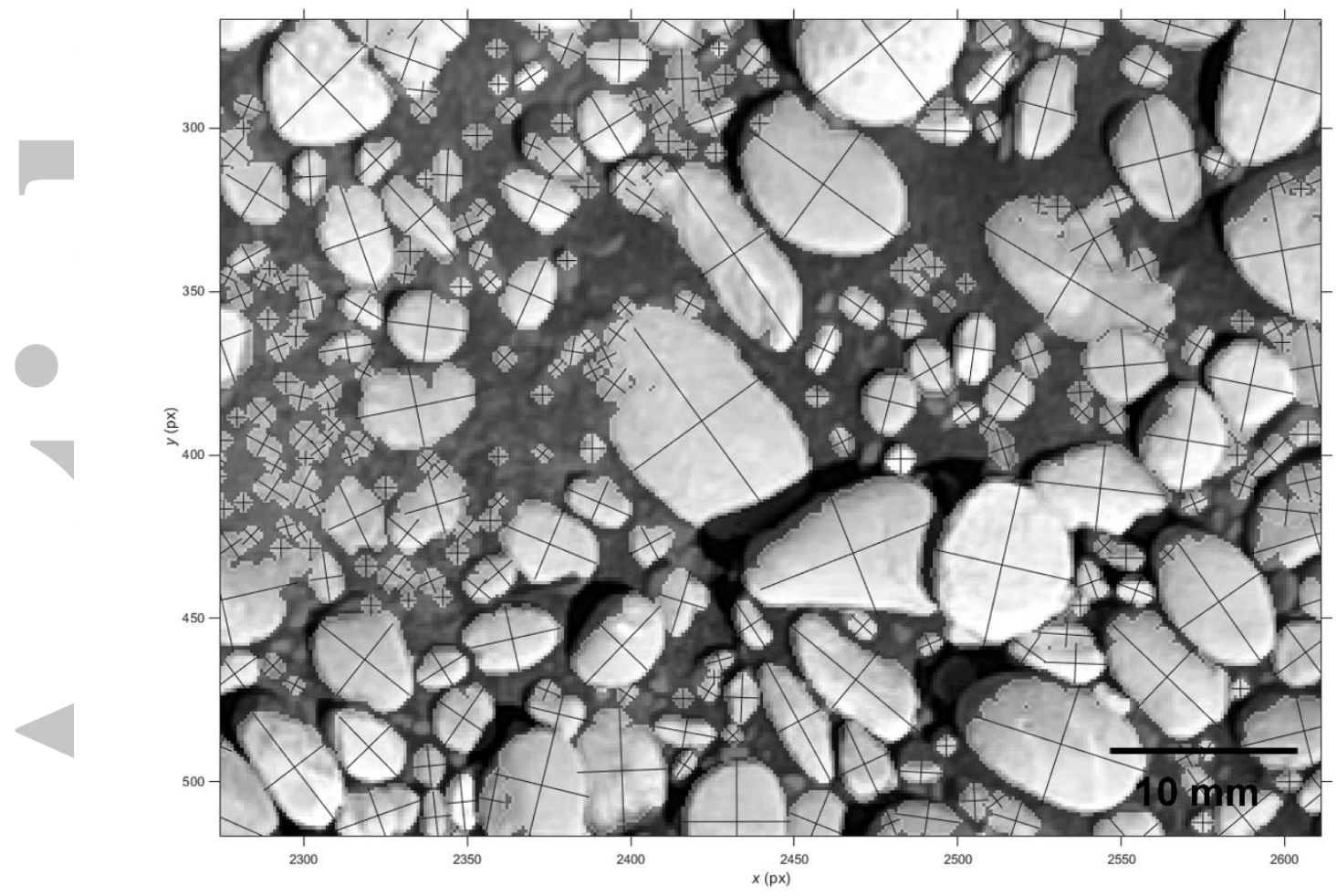

Figure 5. Example of a BASEGRAIN automated photosieving output.

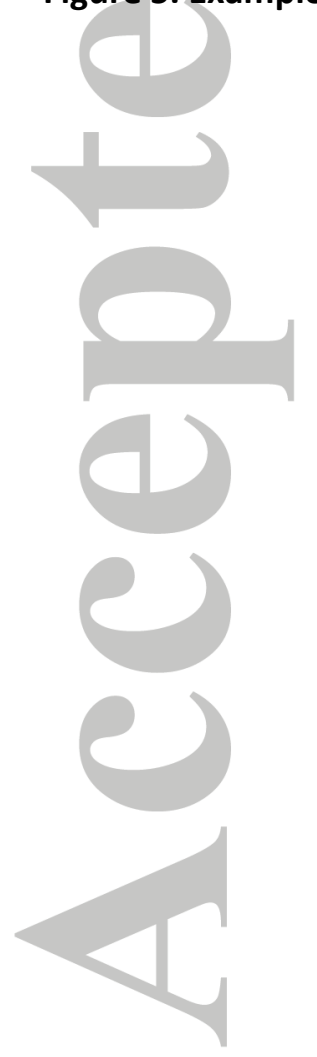

This article is protected by copyright. All rights reserved. 

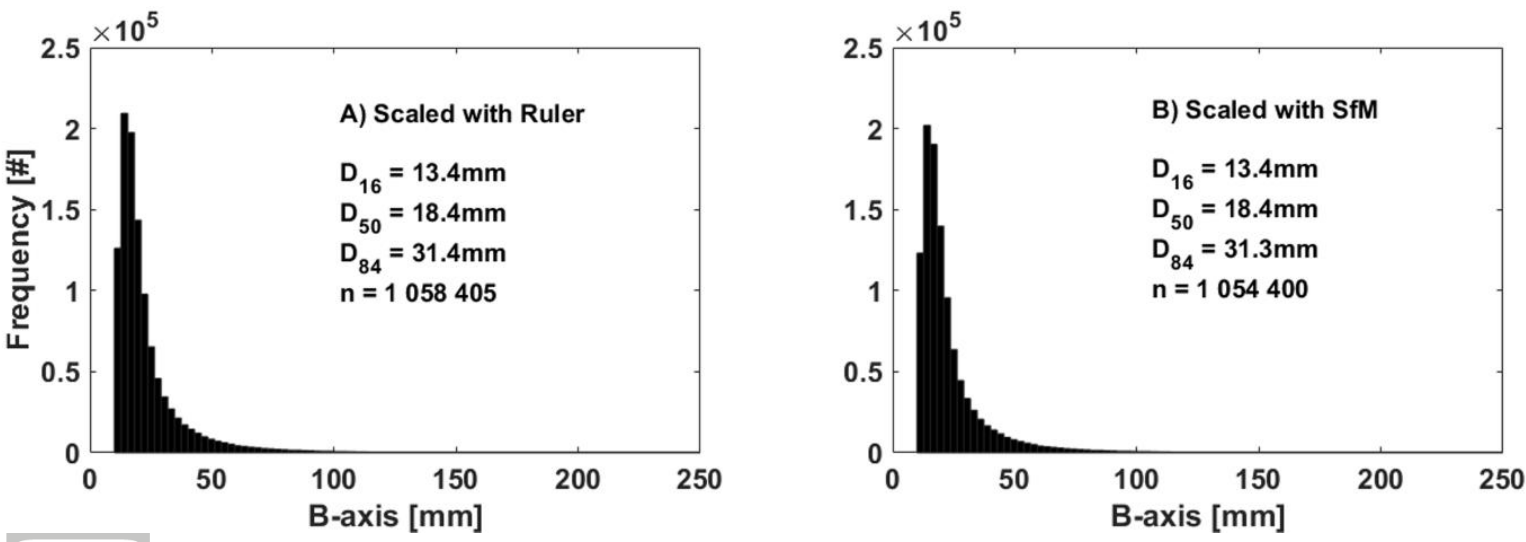

Figure 6. Comparative distributions of photosieving outputs. In part A), the outputs were scaled with manual measurement of the ruler on-screen. In part B), the scale was derived from the SfM workflow described in the paper. A Wilcoxon rank sum test confirmed that the medians of both distributions are statistically equal.
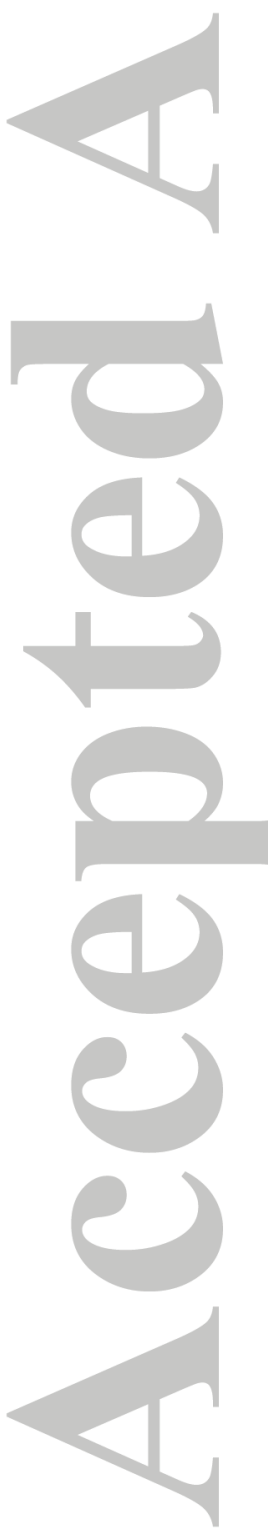

This article is protected by copyright. All rights reserved. 


\section{Robotic photosieving from low-cost multirotor sUAS: A proof-of-concept}

\section{P.E. Carbonnneau*1, S. Bizzi ${ }^{2}$ and G. Marchetti ${ }^{2}$}

1: Department of Geography, Durham University, Durham, UK. Email:

patrice.carbonneau@durham.ac.uk

2: Department of Electronics, Information, and Bioengineering, Politecnico di Milano, Piazza Leonardo da Vinci, Milano, Italy.

*: Corresponding author

\section{Graphical Abstract}

We present a novel approach to grain size sampling: robotic photosieving. Our approach uses drones to collet near-ground imagery. At altitudes below 10 meters, individual clasts are visible in imagery thus opening the path for automated photosieving methods. We show how the additional use of a recently developed workflow for the direct georeferencing of drone-based photogrammetric surveys allows robotic photosieving to operate remotely without the need for any ground validation or direct site access.
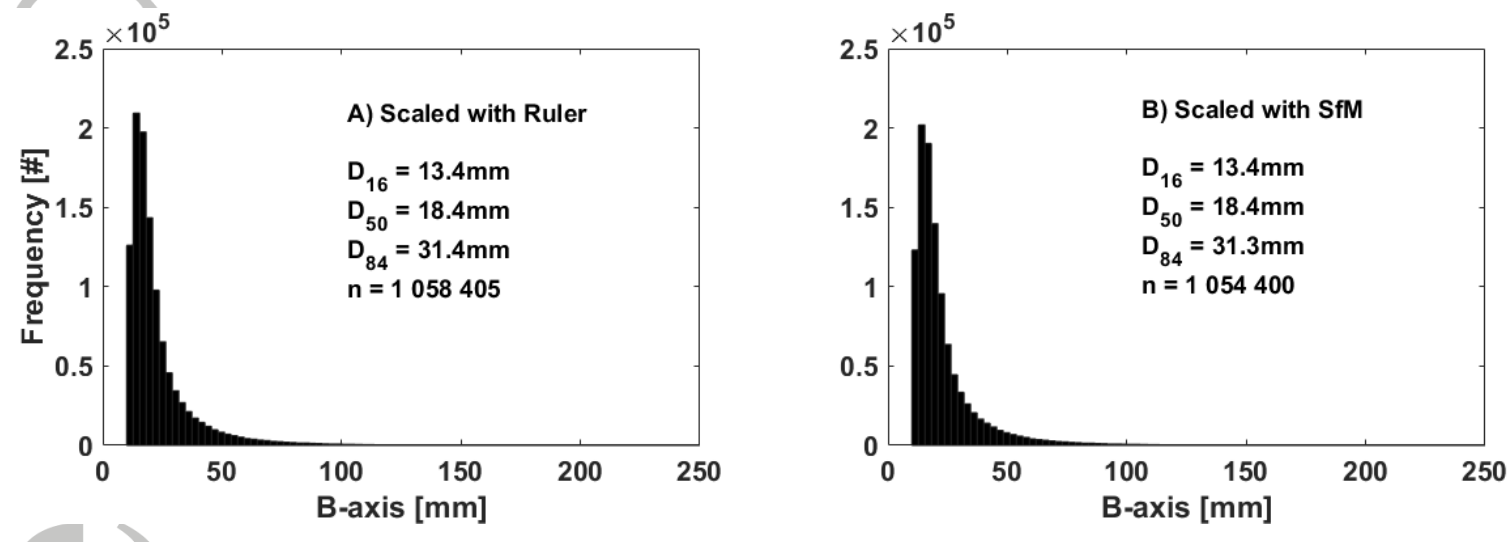

Comparative distributions of robotic photosieving outputs. In part A), the outputs were scaled with manual measurement of the ruler on-screen. In part B), the scale was derived from the SfM workflow described in the paper. A Wilcoxon rank sum test confirmed that the medians of both distributions are statistically equal.

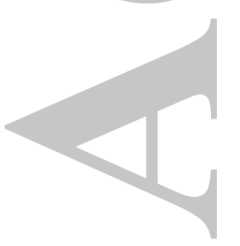

This article is protected by copyright. All rights reserved. 